\title{
Article
}

\section{Impact of Eukaryotic Enteric Pathogens on Malian Children's Gut Mycobiota}

\author{
Aly Kodio 1,2, Estelle Menu 1,2, Safiatou Doumbo ${ }^{3}$, Drissa Coulibaly ${ }^{3}$, \\ Abdoulaye Kassoum Koné ${ }^{3}$, Salimata Konaté ${ }^{3}$, Lamine Tall ${ }^{3,4}$, Ogobara K. Doumbo 3 ,a, \\ Didier Raoult ${ }^{2,4}$, Mahamadou Aly Thera ${ }^{3}$ and Stéphane Ranque ${ }^{1,2, *}$ \\ 1 Aix Marseille Université, Institut de Recherche pour le Développement, Assistance Publique-Hôpitaux de \\ Marseille, Service de Santé des Armées, VITROME : Vecteurs - Infections Tropicales et Méditerranéennes, \\ 19-21 Boulevard Jean Moulin, 13005 Marseille, France; alykodio81@gmail.com (A.K.); \\ estelle.menu@ap-hm.fr (E.M.); stephane.ranque@univ-amu.fr (S.R.) \\ 2 IHU Méditerranée Infection, 19-21 Bd Jean Moulin, 13005 Marseille, France; \\ didier.raoult@mediterranee-infection.com (D.R.) \\ 3 Malaria Research and Training Centre-International Center for Excellence in Research (MRTC-ICER), \\ Department of Epidemiology of Parasitic Diseases, Faculty of Medicine and Dentistry, Université des \\ Sciences, des Techniques et des Technologies de Bamako, Point G, BP 1805 Bamako, Mali; \\ sdoumbo@icermali.org (S.D.); coulibalyd@icermali.org (D.C.); fankone@icermali.org (A.K.K.); \\ sakonate@icermali.org (S.K.); mthera@icermali.org (M.A.T.) \\ 4 Aix Marseille Université, Institut de Recherche pour le Développement, Assistance Publique-Hôpitaux de \\ Marseille, Service de Santé des Armées, MEPHI : Microbes, Evolution, Phylogénie et Infection, 19-21 \\ Boulevard Jean Moulin, 13005 Marseille, France laminetall30@gmail.com (LT) \\ a Deceased. \\ * Correspondence: stephane.ranque@univ-amu.fr
}

\begin{abstract}
Eukaryotic enteric pathogens (EEP) are a public health issue in tropical areas. Yet, their interactions with the gut mycobiota remain poorly understood. We conducted a cross-sectional study in Malian children to analyze the impact of EEP on the gut fungal community. EEP were assessed by qPCR and the gut mycobiota was characterized by ITS1-2 metabarcoding in stool samples collected from 296 children. The 100 controls, in whom no EEP was detected, were compared to: a) 196 children with $\geq 1$ EEP; b) 91 with only Blastocystis; c) 35 with only Giardia intestinalis; and d) 12 with another ( $<1 \%$ each) EPP. The gut fungal community structure was homogenous in each children's group. Linear size-effect discriminant analysis highlighted five relatively more abundant species, including Fusarium longipes and Penicillium caseifulvum, in children with $\geq 1$ EEP, whereas 28, including Aspergillus sydowii and Microdochium colombiense were more abundant in controls. Fusarium, Pyxidiophora, and Stereum abundance was higher in Blastocystis-infected children, whereas Ogataea and Allocryptovalsa were more abundant in controls. Sordariales and Mortierellales abundance was higher in Giardia intestinalis-infected children, whereas Agaricales and Capnodiales abundance was higher in controls. In conclusion, EEP do not significantly alter the gut fungal community structure, and further studies are warranted to confirm our findings that particular taxa are associated with susceptibility or resistance to specific EEP.
\end{abstract}

Keywords: fungal community, gut mycobiota, diversity, ITS metabarcoding, intestinal parasites, children, Mali.

\section{Introduction}

Eukaryotic enteric pathogens (EEP) include protozoa, microsporidia (intracellular parasitic fungi), and helminths, involved in enteric infections. These enteric parasitic infections cause intestinal pain, diarrhea, malabsorption, weight loss. In children living in developing countries, they can also cause chronic infections that are associated with stunting (low height for age), wasting (low 
weight for height), and cognitive impairment [1-7]. Among EEP, Giardia intestinalis infection can cure spontaneously but causes frequent relapses in endemic areas [1]; Blastocystis, another enteric protozoon, is more common in healthy people [8]. Whereas EEP pose a major public health issue in developing countries [9], most of them are included among neglected tropical diseases [10]. The national intestinal parasites control program in Mali relies on water, sanitation, and hygiene measures, combined with broad-spectrum anthelminthic chemoprophylaxis [11]. EEP diagnosis has recently been enhanced by the deployment of DNA-detection based technologies, including real-time PCR and next-generation sequencing [12]. Moreover, metagenomic approaches have been used to examine the influence of EEP on the gut bacterial community [13-15]. Hence, studies in mice have shown that Giardia intestinalis colonization of the small intestine leads to dysbiosis of both aerobic and anaerobic gut bacterial communities, identified by an increase in Proteobacteria and a decrease in Firmicutes and Melainabacteria [16]. Whereas its mechanisms remains controversial, both the sensitivity and severity of Giardia infection in mice were associated with gut bacterial community alterations [6,17]. A growing body of evidence indicate that commensal bacteria community contribute to host health by resisting pathogens, maintaining homeostasis and modulating the immune system [18]. In contrast, data are scarce and little research is focused on the interaction between EEP infection and the gut fungal community. This study aimed to explore the influences of EEP infection on the gut fungal community in healthy Malian children.

\section{Patients and Methods}

Stool samples were obtained from 296 healthy children aged 0-15 years included in a cohort study conducted at the BMP Research Clinic in Bandiagara Health Centre in Mali. The stools were characterized according to the Bristol scale [19]. The solid stools were diluted in equal volume with PBS 10X (phosphate buffered saline solution of pH 7.4, DNase-RNase-free) and all stools collected in sealed vials were stored at $-20^{\circ} \mathrm{C}$.

DNA extraction. The stool DNA was extracted on the EZ1 Advanced XL (QIAGEN Instruments Hombrechtikon, Switzerland) automate with the DNA card bacteria V 1.066069118 QIAGEN using EZ1 DNA tissue kit (Qiagen, France), and eluted in $200 \mu \mathrm{l}$, as previously described [19].

Eukaryotic enteric pathogens qPCR assays. The presence in stool samples of 20 EEP was tested by qPCR, using the primers and probes described in [20], and Master Mix (Roche diagnostics GmbH, Mannheim, Germany) on the CFX96TM or CFX384TM Real-Time PCR Detection Systems (BIO-RAD, Life Science, Marnes-la-coquette, France), as previously described [21].

Fungal metabarcoding. The ITS1 and ITS2 regions amplification products were sequenced on an Illumina MiSeqTM platform at IHU Méditerranée Infection, Marseille, France. The reads and the taxonomic assignment of OTUs were obtained via the PIPITS automated pipeline for the analysis of fungal ITS (internal transcribed spacer) rRNA gene sequences. DNA extraction, amplification, sequencing procedures, and bioinformatics analysis have been detailed in (Kodio et al. 2020).

Statistical analysis. The study design is illustrated in Figure 1. Children were grouped according to their EEP diagnosis results. Those in whom no EEP was detected were considered as controls. Those in whom at least one EEP was detected were defined as cases and compared to the controls. Then, to more precisely analyze the impact of a single EEP infection, the children in whom only one the respective EEP was found: Blastocystis, Giardia intestinalis, and other EEPs were compared to the controls. The gut fungal community structure was described for each group of children via the Shannon $\mathrm{H}$ and Chao- 1 indices, their median value and interquartile range was calculated using the GraphPad Prism 5.03 software and the Mann Whitney U test was used to compare the groups. Infected and non-infected children's gut community structure were compared via Coordinated Principal Component Analysis (PCoA) and PerMANOVA (Permutational MANOVA) test based on the Bray-Curtis similarity index with the PAST 4.01 software [22]. The differential distribution of taxa between the infected children's groups and controls was assessed using linear size effect discriminant analysis (LEfSe) [23]. Two-sided $\mathrm{P}$ values $<0.05$ were considered statistically significant. 
This study protocol was approved by the Ethics Committee of the Faculty of Medicine of Mali (N²017/133/CE/FMPOS). Each child included and at least one of his or her parents or the person in charge gave their informed written consent to participate in the study.

\section{Results}

\subsection{Characteristics of the studied children}

The mean age of the 296 children was 7.6 years (95\% CI [7.1 - 8.0]), 153 (51.7\%) were female. At least one EEP was detected in 196 (66.2\%) of the children, including Blastocystis in 149 (49.3\%), Giardia intestinalis, in 87 (29.4\%), Enterocytozoon bieneusi in 10 (3.4\%), Entamoeba histolytica, Dientamoeba fragilis and Trichuris trichiura each in 3 (1.4\%) (Table 1; Figure 1). Encephalitozoon intestinalis, Cyclospora cayetanensis, Cryptosporidium parvum, Ancylostoma duodenale, Ascaris lumbricoides, Hymenolepis diminuta, Necator americanus, Strongyloides stercoralis, Taenia solium, Taenia saginata, Enterobius vermicularis were detected in none of the children. None of the EEP tested was detected in controls.

Table 1. Prevalence of eukaryotic enteric pathogens detected by qPCR in 296 healthy Malian children.

\begin{tabular}{ccc}
\hline Eukaryotic enteric pathogens & $\mathbf{N}$ & Prevalence (\%) \\
\hline Blastocystis & 147 & 49.7 \\
Giardia intestinalis & 87 & 29.4 \\
Enterocytozoon bieneusi & 10 & 3.4 \\
Entamoeba histolytica & 4 & 1.3 \\
Dientamoeba fragilis & 3 & 1.0 \\
Trichuris trichiura & 3 & 1.0 \\
Balantidium coli & 1 & 0.3 \\
Isospora belli & 1 & 0.3 \\
Schistosoma mansoni & 1 & 0.3 \\
\hline
\end{tabular}

\subsection{Eukaryotic enteric pathogens distribution}

The study design is described in Figure 1, briefly the $(n=100)$ healthy control were compared to four groups of children: 1) 196 with at least one EEP detected; 2) 91 in whom only Blastocystis was detected; 3) 35 in whom only Giardia intestinalis was detected; and 4) 12 in whom only one of the following EEP was detected: Enterocytozoon bieneusi ( $\mathrm{n}=7)$, Entamoeba histolytica $(\mathrm{n}=3)$, Dientamoeba fragilis $(n=1)$, Cyclospora cayetanensis $(n=1)$. The characteristics of the children are detailed in Table 2. 
4 of 10

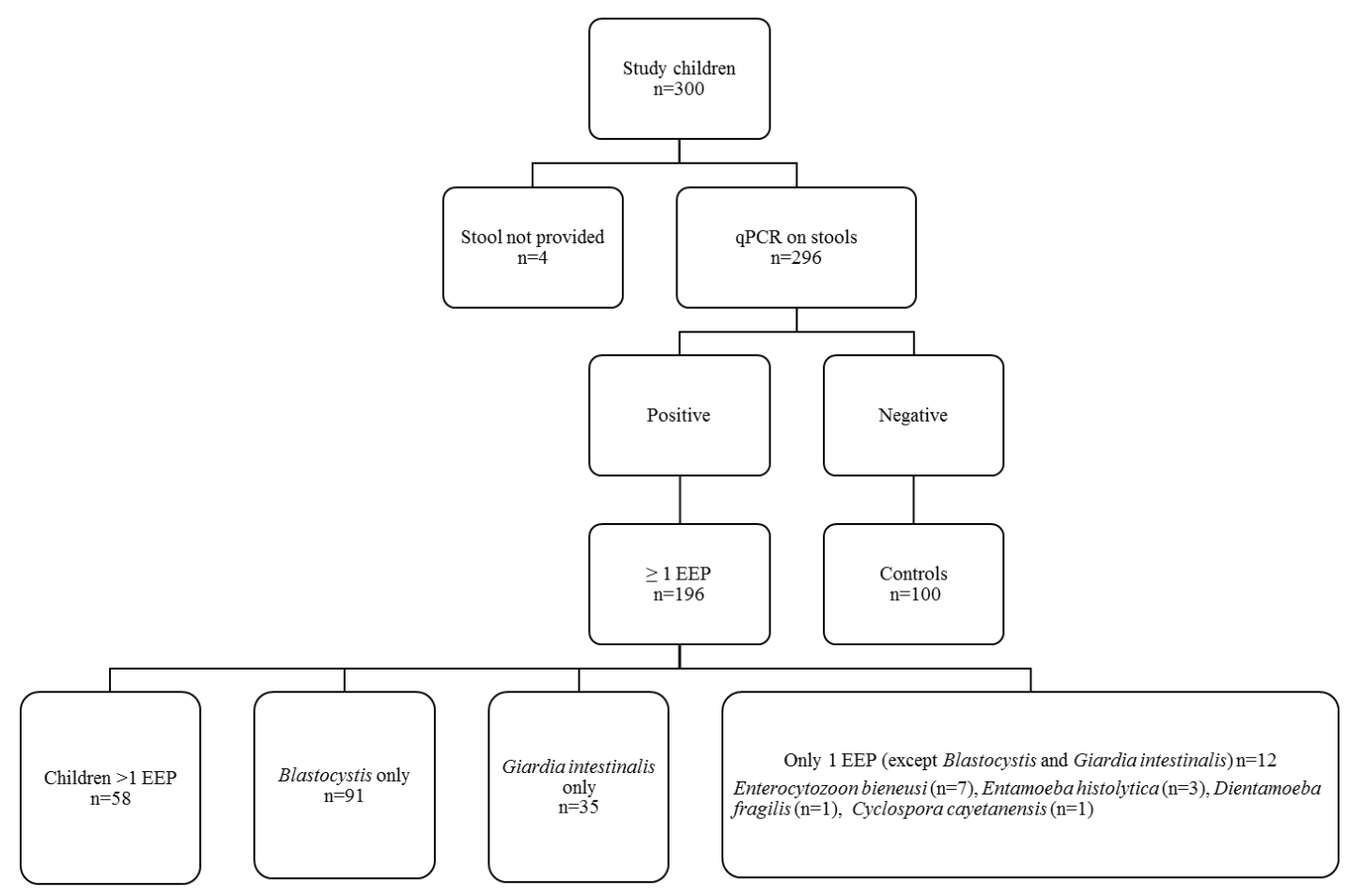

Figure 1. Study flow chart. (EEP: eukaryotic enteric pathogen.). 
Table 2. Gut fungi community structure (median and interquartile ranges of Shannon H diversity and Chao 1 species richness indices) in controls and children with enteric eukaryotic pathogens (EEP).

\begin{tabular}{|c|c|c|c|c|c|}
\hline Median [IQR] (P value) & Controls $(n=100)$ & Any EEP (n=196) & Blastocystis $(n=91)$ & Giardia intestinalis $(n=35)$ & Others $^{1}(\mathrm{n}=12)$ \\
\hline entry 1 & data & & & & data \\
\hline Age (years) & $7[3-10.6]$ & $8[5-11](P=0.08)^{2}$ & $9[6-11](P=0.01)$ & $7[5-10](P=0.99)$ & $6[1.3-10.8](P=0.44)$ \\
\hline Males (\%) & 45 & $50(\mathrm{P}=0.461)$ & $45.1(\mathrm{P}=1.00)$ & $48.6(\mathrm{P}=0.84)$ & $58.3(\mathrm{P}=0.54)$ \\
\hline Weight (kg) & $21[12.9-30.5]$ & $24[17.7-33.4](P=0.04)$ & $26.6[19-35.7](\mathrm{P}=0.01)$ & $20.85[17.58-33.85](\mathrm{P}=0.56)$ & $13.5[9.5-34.3](\mathrm{P}=0.20)$ \\
\hline Height (cm) & 124 [95 - 139.5] & $125[110-142](\mathrm{P} 0.23)$ & $129[114.3-146.8](P=0.03)$ & $117.5[108.5-131.3](\mathrm{P}=0.83)$ & $111[65-135](P=0.22)$ \\
\hline Axillary temperature $\left({ }^{\circ} \mathrm{C}\right)$ & $36.35[36-36.7]$ & $36.5[36.1-36.8](\mathrm{P}=0.49)$ & $36.5[36.1-36.7](\mathrm{P}=0.62)$ & $36.70[36-36.8](P=0.20)$ & $36.2[35.9-36.8](P=0.41)$ \\
\hline Hemoglobin level (g/dL) & $12.0[11.2-13.1]$ & $12.7[11.6-13.7](\mathrm{P}<0.01)$ & $12.8[11.7-13.8](\mathrm{P}=0.01)$ & $12.5[11.6-13.5](\mathrm{P}=0.12)$ & $11.1[10.5-13](\mathrm{P}=0.14)$ \\
\hline \multicolumn{6}{|l|}{ Stool characteristics } \\
\hline Hard (\%) & 12 & $12.2(\mathrm{P}=0.74)$ & $8.8(\mathrm{P}=0.49)$ & $22.9(\mathrm{P}=0.07)$ & $25.1(\mathrm{P}=0.20)$ \\
\hline Normal (\%) & 32 & $44.4(\mathrm{P}=0.05)$ & $44(\mathrm{P}=0.10)$ & $31.4(\mathrm{P}=0.96)$ & $33.3(\mathrm{P}=0.10)$ \\
\hline Loose $(\%)$ & 54 & $41.3(\mathrm{P}=0.05)$ & $46.2(\mathrm{P}=0.31)$ & $42.9(\mathrm{P}=0.33)$ & $33.3(\mathrm{P}=0.23)$ \\
\hline Not done (\%) & 2 & $2.1(\mathrm{P}=1.00)$ & $1(\mathrm{P}=0.61)$ & $2.8(\mathrm{P}=0.66)$ & $8.3(\mathrm{P}=0.29)$ \\
\hline Blood (\%) & 1 & $2(\mathrm{P}=0.67)$ & $3.3(\mathrm{P}=0.08)$ & $0(\mathrm{P}=0.70)$ & $8.3(\mathrm{P}=0.20)$ \\
\hline Melena (\%) & 8 & $8.2(\mathrm{P}=0.91)$ & $7.7(\mathrm{P}=0.96)$ & $11.4(\mathrm{P}=0.51)$ & $16.7(\mathrm{P}=0.30)$ \\
\hline \multicolumn{6}{|c|}{ Gut fungal community structures } \\
\hline Chao-1 & $75.9[52.5-99.1]$ & $74.1[55.1-92.8](\mathrm{P}=0.73)$ & $71.5[54-90.9](\mathrm{P}=0.38)$ & $75.9[52.5-99.1](\mathrm{P}=0.71)$ & $67.7[54.6-80.8](\mathrm{P}=0.34)$ \\
\hline
\end{tabular}

${ }^{2}$ Each group of children with EEP was compared to controls by using the Mann Whitney U or the Fischer exact tests as appropriate. 
6 of 10

\subsection{Gut fungal microbiota}

The children's fungal gut community structure was assessed via ITS1 and ITS2 metabarcoding on an Illumina MiSeq ${ }^{\mathrm{TM}}$ platform, as described in (Kodio et al. 2020). Briefly, the ITS1 region analysis yielded 647816 reads and 532 single OTUs; the ITS2 region yielded 1975320 reads and 479 single OTUs. Among the 645 detected fungal taxa, 323 were identified via ITS1 reads, 224 via ITS2 reads, and 98 via both ITS1 and ITS2 reads. The ITS1 and ITS2 OTU tables were combined for further analyses. The phyla were distributed as follows: Ascomycota (35.5\%), Basidiomycota $(35.3 \%)$, Chytridiomycota (0.2\%), Mortierellomycota (0.1\%), Mucoromycota (1.2\%), and Unclassified (16.3\%). Of note Plantae Streptophyta accounted to $11.4 \%$ of the OTU (mainly ITS2 region). The age group (0 - 5) included all phyla. Abundance of phyla was highest in the age group $(0-5)$ followed by the age group $(6-10)$ and the age group (11 - 15). The frequency of phyla was highest in the age group $(6-$ $10)$ followed by the age group $(0-5)$ and the group $(11-15)$.

\subsection{Colonization of intestinal parasites in children and diversity of fungal digestive microbiota}

PCoA based on the OTU Bray-Curtis similarity index is illustrated for at least one EEP (Figure 2A); Blastocystis (Figure 2B); Giardia intestinalis (Figure 2C), and other parasites (one of each) (Figure 2D). Heterogeneity between the fungal communities of each group of children and those of the control group of children was not statistically significant with the PERMANOVA (Permutational MANOVA) test. Alpha diversity analysis showed that the gut fungal community structure, assessed via diversity (Shannon $\mathrm{H}$ ) and species richness (Chao-1) were homogeneous between children with at least one EEP or any of the EEP infected children groups and controls (Table 2).

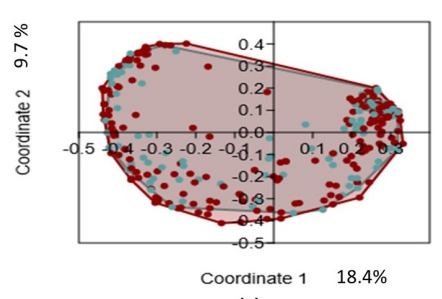

(a)

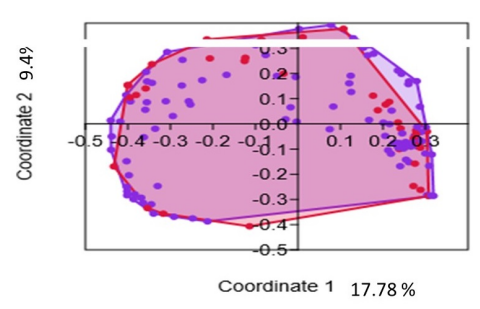

(c)

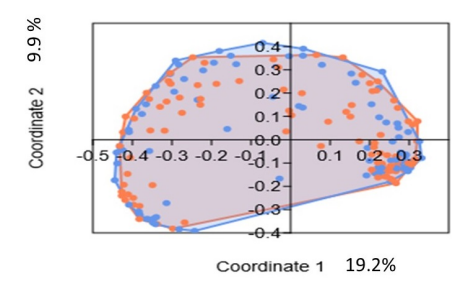

(b)

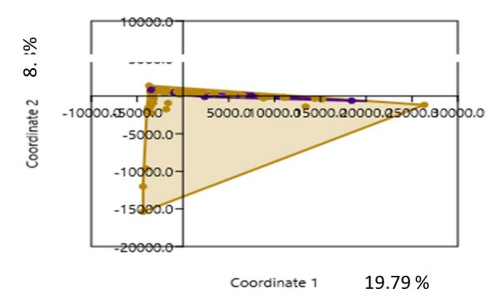

(d)

Figure 2. A Bray-Curtis similarity index based Principal Component Analysis of the gut fungal community in children illustrating the similarities between the gut fungal community of children with eukaryotic enteric pathogens (EEP) and controls (with no EEP). Heterogeneity between fungal communities was tested with PERMANOVA in children with: (a) at least one EEP ( $\mathrm{P}=0.63)$; (b) Blastocystis only $(\mathrm{P}=0.59)$; (c) Giardia intestinalis only $(\mathrm{P}=0.36)$; and $(\mathrm{d})$ other parasites (one of each) $(\mathrm{P}=0.63)$. 


\subsection{Fungal community structure associated with eukaryotic enteric pathogens}

Size-Effect Linear Discriminant Analysis (LEfSe) was used to test which fungal taxa were relatively more or less abundant in each of the children with EEP group compared to the controls. When comparing children with at least one EEP with controls, operational taxonomic unit (OTU) assigned at the Order and Family are detailed in Figure S1A-S1B. At genus level, Papiliotrema, Zygosporium were more abundant in children with at least one EEP and Microdochium, Fusicolla, Trichaptum, Tricholoma, Parasarocladium were more abundant in controls (Figure 1C); five fungal species, including Fusarium longipes, Penicillium caseilulvum were more abundant in children with at least one EEP and 28 species with Aspergillus sydowii, Microdochium colombiense were more abundant in controls (Figure S1D). When comparing children with Blastocystis carriage with controls, among fungal OTU assigned to the Class level Laboulbeniomycetes and Leotiomycetes were relatively more abundant in children with Blastocystis and controls, respectively (Figure S2A). Fungal OTU assigned at the Order and Family levels are detailed in Figure S2B, S2C. At the genus level, Fusarium, Pyxidiophora, Stereum, Alternaria, Papiliotrema, Bulleria, Ustilago, Pseudofusicoccum, Unclassified Pezizaceae, and Edenia were more abundant in children with Blastocystis, whereas Ogataea, Allocryptovalsa were more abundant in control (Figure S2D). And at the species level, LEfSe identified 16 fungal taxa that were more abundant in children with Blastocystis, whereas nine were relatively more abundant in controls (Figure S2E).

When comparing children with Giardia intestinalis carriage with controls, among fungal OTU assigned to the Class level, Mortierellomycetes and Eurotiomycetes were relatively more abundant in children with Giardia and controls, respectively (Figure S3A). Fungal OTU assigned at the Order and Family levels are detailed in Figure S3B, S3C. Twenty-three fungal genera and 14 species were relatively more abundant in children with Giardia, whereas seven fungal genera and seven species were relatively more abundant in controls (Figure S3D, S3E). The comparisons of children carrying other (mixed) EEP with controls are detailed in Figures S4A-S4E.

\section{Discussion}

To the best of our knowledge, this is the first study where ITS1-2 Illumina MiSeq ${ }^{\circledR}$ metabarcoding was used to characterize the gut fungal community associated with EEP. Metabarcoding of 296 stool samples allowed the detection of 532 fungal species using the ITS1 barcode, 479 fungal species using the ITS2 barcode, and only 98 fungal species detected with both barcodes. Our finding contrast with those of another study where the total number of OTU in the ITS2 region exceeded that of the ITS1 region [24].

Among this study limitations, our finding in Malian children living in Bandiagara cannot be extrapolated to other populations. Moreover, the proportion of Blastocystis carriage was high and explains the relatively similar factors that were associated with the carriage of at least one EEP or Blastocystis only (Table 2). Also, the 12 children sample who were infected by Enterocytozoon bieneusi, Entamoeba histolytica, Dientamoeba fragilis, or Cyclospora cayetanensis was too small to allow drawing robust conclusions. Furthermore, we found no other study addressing the complex interactions between EEP and the fungal gut community, and the prevalence of many EEP, and particularly helminths, was quite low in this study, probably due to the current broad-spectrum anthelminthic chemoprophylaxis policy in children in Mali. Therefore, this study findings should be interpreted with caution and should be replicated in both similar and distinct populations to strengthen our conclusions.

The data on EEP-fungi interactions remain scarce. The co-incubation with Candida albicans and Candida glabrata has been shown to reduce Blastocystis growth [25]. Also, Saccharomyces boulardii has displayed beneficial effect on symptomatic Blastocystis infection [26]. Previous results have shown that Blastocystis colonization did not alter the diversity and richness of the gut fungal community in Malian children. This contrasts with its association with a higher diversity of the gut bacterial communities in the same population [20]. Some fungal taxa were associated with the presence or absence of Blastocystis in children. Interestingly, an entomo- and phytopathogenic Fusarium sp., a 
xerophilic Aspergillus section Restricti [27], a wood decaying Coriticiaceae, and an arthropod-associated Pyxidiophora sp. were associated with Blastocystis colonization, whereas the relatively common human opportunistic pathogenic yeast Candida tropicalis was relatively more abundant in non-colonized controls. Similarly, the presence of Giardia intestinalis was not associated with alteration of the gut fungal community structure. Yet, species including Fusarium longipes Penicillium freii, Stereum hirsutum, and again Candida tropicalis, were relatively abundant in controls children free from Giardia. The fungal chitosan prepared from the cell walls of Penicillium viridicatum and Penicillium aurantiogriseum has been found effective against Giardia intestinalis cysts [28]. In another study, Saccharomyces boulardii showed a potential benefit for the treatment of acute gastroenteritis in paediatric patients [29]. More interestingly, Blastocystis is usually associated with a "healthy" gut microbiota whereas Giardia is usually associated with gut dysbiosis [29,30]. Thus, whether the wood decaying Coriticiaceae Stereum hirsutum, or the common human opportunistic pathogenic yeast Candida tropicalis are associated with a dysbiotic or a healthy gut microbiota warrant further studies.

\section{Conclusions}

This study provides new insights into the complex interactions between the gut fungal community and EEP infection/colonisation. We found that particular fungal taxa abundance differed significantly between children carrying common EEP and controls. To date answering the questions whether these are cause-and-effect relationships and whether particular gut microbiota fungal taxa are associated with susceptibility/resistance to specific EEP warrant further studies.

Supplementary Materials: Figure S1: Comparison of the fungal digestive microbiota between children infected with at least one parasite and controls. The linear size effect discriminant analysis (LEfSe) was performed considering score 2 and the Kruskal-Wallis test at 0.05. A) Differentiation of fungus Orders between children with at least one parasite (green) and control (red); B) At Family level; C) At genus level and D) Species level, Figure S2: The linear size effect discriminant analysis (LEfSe) comparison of the gut fungi communities in children infected with Blastocystis (green bars) and healthy control (red bars) children, at the A) Phylum; B) Order; C) Family; D) genus; and E) species levels. (The LEfSe was performed with score $>2$ and the Kruskal-Wallis test $\mathrm{P}<0.05$ thresholds), Figure S3: The linear size effect discriminant analysis (LEfSe) comparison of the gut fungi communities in children infected with Giardia intestinalis (green bars) and healthy control (red bars) children, at the A) Orders; B) Family; C) genus; and D) species levels. (The LEfSe was performed with score $>2$ and the Kruskal-Wallis test $\mathrm{P}<0.05$ thresholds). Figure S4: The linear size effect discriminant analysis (LEfSe) comparison of the gut fungi communities in children infected (green bars) with one of the following eukaryotic enteric pathogen (Enterocytozoon bieneusi $(\mathrm{n}=7)$, Entamoeba histolytica $(\mathrm{n}=3)$, Dientamoeba fragilis $(\mathrm{n}=1)$, Cyclospora cayetanensis $(\mathrm{n}=1)$ ) and healthy control (red bars) children, at the A) Phylum; B) Order; C) Family; D) genus; and E) species levels. (The LEfSe was performed with score $>2$ and the Kruskal-Wallis test $\mathrm{P}<0.05$ thresholds).

Author Contributions: O.K. Doumbo, D. Raoult, S. Ranque and A. Kodio designed and conceived the study. D. Coulibaly, A.K. Koné, S. Doumbo, and M.A. Théra included the study participants, and performed clinical and biological evaluations, sample collection and data management. A. Kodio, and E. Menu performed the laboratory work. A. Kodio, L. Tall, S. Ranque did the bioinformatics and statistical analysis. The manuscript was drafted by A. Kodio and edited by S. Ranque. All authors have read and agreed to the published version of the manuscript.

Funding: This research was funded by the French Government under the 'Investissements d'avenir' (Investments for the Future) programme managed by the Agence Nationale de la Recherche (ANR, fr: National Agency for Research), [reference: Méditerranée Infection 10-IAHU-03], the IHU-Mediterranean Infection Foundation, and the MARCAD DELTAS Africa Initiative grant DEL-15-10. The DELTAS Africa Initiative is an independent funding scheme of the African Academy of Sciences' (AAS) Alliance for Accelerating Excellence in Science in Africa (AESA) and supported by the New Partnership for Africa's Development Planning and Coordinating Agency (NEPAD Agency) with funding from the Wellcome Trust grant 107741/A/15/Z and the UK government. The views expressed in this publication are those of the author(s) and not necessarily those of the AAS, the NEPAD Agency, the Wellcome Trust, or the UK government. 
Acknowledgments: The authors would like to thank the authorities and the people of Bandiagara for their participation in the study; and the IHU MI sequencing platform team, especially Enora Tomeï, for their technical assistance for the ITS1-ITS2 Illumina MiSeq metagenomics analyses.

Conflicts of Interest: The authors declare no conflict of interest.

\section{References}

1. Squire, S.A.; Ryan, U. Cryptosporidium and Giardia in Africa: current and future challenges. Parasit. Vectors 2017, 10, doi:10.1186/s13071-017-2111-y.

2. Berkman, D.S.; Lescano, A.G.; Gilman, R.H.; Lopez, S.L.; Black, M.M. Effects of stunting, diarrhoeal disease, and parasitic infection during infancy on cognition in late childhood: a follow-up study. The Lancet 2002, 359, 564-571, doi:10.1016/S0140-6736(02)07744-9.

3. Halliez, M.C.M.; Buret, A.G. Extra-intestinal and long term consequences of Giardia duodenalis infections. World J. Gastroenterol. 2013, 19, 8974-8985, doi:10.3748/wjg.v19.i47.8974.

4. Halliez, M.C.M.; Motta, J.-P.; Feener, T.D.; Guérin, G.; LeGoff, L.; François, A.; Colasse, E.; Favennec, L.; Gargala, G.; Lapointe, T.K.; et al. Giardia duodenalis induces paracellular bacterial translocation and causes postinfectious visceral hypersensitivity. Am. J. Physiol. - Gastrointest. Liver Physiol. 2016, 310, G574-G585, doi:10.1152/ajpgi.00144.2015.

5. Nematian, J.; Gholamrezanezhad, A.; Nematian, E. Giardiasis and other intestinal parasitic infections in relation to anthropometric indicators of malnutrition: a large, population-based survey of schoolchildren in Tehran. Ann. Trop. Med. Parasitol. 2008, 102, 209-214, doi:10.1179/136485908X267876.

6. Allain, T.; Amat, C.B.; Motta, J.-P.; Manko, A.; Buret, A.G. Interactions of Giardia sp. with the intestinal barrier: Epithelium, mucus, and microbiota. Tissue Barriers 2017, 5, doi:10.1080/21688370.2016.1274354.

7. Al-Mekhlafi, H.M.; Al-Maktari, M.T.; Jani, R.; Ahmed, A.; Anuar, T.S.; Moktar, N.; Mahdy, M.A.K.; Lim, Y.A.L.; Mahmud, R.; Surin, J. Burden of Giardia duodenalis Infection and Its Adverse Effects on Growth of Schoolchildren in Rural Malaysia. PLoS Negl. Trop. Dis. 2013, 7, doi:10.1371/journal.pntd.0002516.

8. Mardani Kataki, M.; Tavalla, M.; Beiromvand, M. Higher prevalence of Blastocystis hominis in healthy individuals than patients with gastrointestinal symptoms from Ahvaz, southwestern Iran. Comp. Immunol. Microbiol. Infect. Dis. 2019, 65, 160-164, doi:10.1016/j.cimid.2019.05.018.

9. Jong, E. Intestinal parasites. Prim. Care Clin. Off. Pract. 2002, 29, 857-877, doi:10.1016/S0095-4543(02)00047-7.

10. Savioli, L.; Smith, H.; Thompson, A. Giardia and Cryptosporidium join the 'Neglected Diseases Initiative.' Trends Parasitol. 2006, 22, 203-208, doi:10.1016/j.pt.2006.02.015.

11. Dabo, A.; Badawi, H.M.; Bary, B.; Doumbo, O.K. Urinary schistosomiasis among preschool-aged children in Sahelian rural communities in Mali. Parasit. Vectors 2011, 4, doi:10.1186/1756-3305-4-21.

12. Verweij, J.J.; Stensvold, C.R. Molecular testing for clinical diagnosis and epidemiological investigations of intestinal parasitic infections. Clin. Microbiol. Rev. 2014, 27, 371-418, doi:10.1128/CMR.00122-13.

13. Nieves-Ramírez, M.E.; Partida-Rodríguez, O.; Laforest-Lapointe, I.; Reynolds, L.A.; Brown, E.M.; Valdez-Salazar, A.; Morán-Silva, P.; Rojas-Velázquez, L.; Morien, E.; Parfrey, L.W.; et al. Asymptomatic Intestinal Colonization with Protist Blastocystis Is Strongly Associated with Distinct Microbiome Ecological Patterns. mSystems 2018, 3, e00007-18, doi:10.1128/mSystems.00007-18.

14. Torres, M.F.; Uetanabaro, A.P.T.; Costa, A.F.; Alves, C.A.; Farias, L.M.; Bambirra, E.A.; Penna, F.J.; Vieira, E.C.; Nicoli, J.R. Influence of bacteria from the duodenal microbiota of patients with symptomatic giardiasis on the pathogenicity of Giardia duodenalis in gnotoxenic mice. J. Med. Microbiol. 2000, 49, 209-215, doi:10.1099/0022-1317-49-3-209.

15. Cattadori, I.M.; Sebastian, A.; Hao, H.; Katani, R.; Albert, I.; Eilertson, K.E.; Kapur, V.; Pathak, A.; Mitchell, S. Impact of Helminth Infections and Nutritional Constraints on the Small Intestine Microbiota. PLOS ONE 2016, 11, e0159770, doi:10.1371/journal.pone.0159770.

16. Barash, N.R.; Maloney, J.G.; Singer, S.M.; Dawson, S.C. Giardia Alters Commensal Microbial Diversity throughout the Murine Gut. Infect. Immun. 2017, 85, doi:10.1128/IAI.00948-16. 
17. Keselman, A.; Li, E.; Maloney, J.; Singer, S.M. The Microbiota Contributes to CD8+ T Cell Activation and Nutrient Malabsorption following Intestinal Infection with Giardia duodenalis. Infect. Immun. 2016, 84, 2853-2860, doi:10.1128/IAI.00348-16.

18. Zhang, Y.; Wang, X.; Li, H.; Ni, C.; Du, Z.; Yan, F. Human oral microbiota and its modulation for oral health. Biomed. Pharmacother. 2018, 99, 883-893, doi:10.1016/j.biopha.2018.01.146.

19. Riegler, G.; Esposito, I. Bristol scale stool form. A still valid help in medical practice and clinical research. Tech. Coloproctology 2001, 5, 163-164, doi:10.1007/s101510100019.

20. Sow, D.; Parola, P.; Sylla, K.; Ndiaye, M.; Delaunay, P.; Halfon, P.; Camiade, S.; Dieng, T.; Tine, R.C.K.; Faye, B.; et al. Performance of Real-Time Polymerase Chain Reaction Assays for the Detection of 20 Gastrointestinal Parasites in Clinical Samples from Senegal. Am. J. Trop. Med. Hyg. 2017, 97, 173-182, doi:10.4269/ajtmh.16-0781.

21. Kodio, A.; Coulibaly, D.; Koné, A.K.; Konaté, S.; Doumbo, S.; Guindo, A.; Bittar, F.; Gouriet, F.; Raoult, D.; Thera, M.A.; et al. Blastocystis Colonization Is Associated with Increased Diversity and Altered Gut Bacterial Communities in Healthy Malian Children. Microorganisms 2019, 7, doi:10.3390/microorganisms7120649.

22. Hammer, O.; Harper, D.A.T.; Ryan, P.D. PAST: Paleontological Statistics Software Package for Education and Data Analysis. Palaeontologia Electronica 2001, 4 1-9.

23. Segata, N.; Izard, J.; Waldron, L.; Gevers, D.; Miropolsky, L.; Garrett, W.S.; Huttenhower, C. Metagenomic biomarker discovery and explanation. Genome Biol. 2011, 12, R60, doi:10.1186/gb-2011-12-6-r60.

24. Hamad, I.; Ranque, S.; Azhar, E.I.; Yasir, M.; Jiman-Fatani, A.A.; Tissot-Dupont, H.; Raoult, D.; Bittar, F. Culturomics and Amplicon-based Metagenomic Approaches for the Study of Fungal Population in Human Gut Microbiota. Sci. Rep. 2017, 7, 16788, doi:10.1038/s41598-017-17132-4.

25. Lepczyńska, M.; Dzika, E. The influence of probiotic bacteria and human gut microorganisms causing opportunistic infections on Blastocystis ST3. Gut Pathog. 2019, 11, doi:10.1186/s13099-019-0287-8.

26. Dinleyici, E.C.; Eren, M.; Dogan, N.; Reyhanioglu, S.; Yargic, Z.A.; Vandenplas, Y. Clinical efficacy of Saccharomyces boulardii or metronidazole in symptomatic children with Blastocystis hominis infection. Parasitol. Res. 2011, 108, 541-545, doi:10.1007/s00436-010-2095-4.

27. Yarahmadi, M.; Fakhar, M.; Ebrahimzadeh, M.A.; Chabra, A.; Rahimi-esboei, B. The anti-giardial effectiveness of fungal and commercial chitosan against Giardia intestinalis cysts in vitro. J. Parasit. Dis. Off. Organ Indian Soc. Parasitol. 2016, 40, 75-80, doi:10.1007/s12639-014-0449-z.

28. Padayachee, M.; Visser, J.; Viljoen, E.; Musekiwa, A.; Blaauw, R. Efficacy and safety of Saccharomyces boulardii in the treatment of acute gastroenteritis in the paediatric population: a systematic review. South Afr. J. Clin. Nutr. 2019, 32, 58-69, doi:10.1080/16070658.2018.1449378.

29. Tito, R.Y.; Chaffron, S.; Caenepeel, C.; Lima-Mendez, G.; Wang, J.; Vieira-Silva, S.; Falony, G.; Hildebrand, F.; Darzi, Y.; Rymenans, L.; et al. Population-level analysis of Blastocystis subtype prevalence and variation in the human gut microbiota. Gut 2019, 68, 1180-1189, doi:10.1136/gutjnl-2018-316106.

30. Berry, A.S.F.; Johnson, K.; Martins, R.; Sullivan, M.C.; Farias Amorim, C.; Putre, A.; Scott, A.; Wang, S.; Lindsay, B.; Baldassano, R.N.; et al. Natural Infection with Giardia Is Associated with Altered Community Structure of the Human and Canine Gut Microbiome. mSphere 2020, 5, doi:10.1128/mSphere.00670-20. 